\title{
An intermediate value theorem for monotone operators in ordered Banach spaces
}

\author{
Vadim Kostrykin ${ }^{1 *}$ and Anna Oleynik ${ }^{1,2}$
}

\author{
"Correspondence: \\ kostrykin@mathematik.uni-mainz.de \\ ${ }^{1}$ FB 08 - Institut für Mathematik, \\ Johannes Gutenberg-Universität \\ Mainz, Staudinger Weg 9, Mainz, \\ D-55099, Germany \\ Full list of author information is \\ available at the end of the article
}

\begin{abstract}
We consider a monotone increasing operator in an ordered Banach space having $u_{-}$ and $u_{+}$as a strong super- and subsolution, respectively. In contrast with the well-studied case $u_{+}<u_{-}$, we suppose that $u_{-}<u_{+}$. Under the assumption that the order cone is normal and minihedral, we prove the existence of a fixed point located in the order interval $\left[u_{-}, u_{+}\right]$.
\end{abstract}

MSC: $47 \mathrm{H} 05 ; 47 \mathrm{H} 10 ; 46 \mathrm{~B} 40$

Keywords: fixed point theorems in ordered Banach spaces

It is an elementary consequence of the intermediate value theorem for continuous realvalued functions $f:\left[a_{1}, a_{2}\right] \rightarrow \mathbb{R}$ that if either

$$
f\left(a_{1}\right)>a_{1} \text { and } f\left(a_{2}\right)<a_{2}
$$

or

$$
f\left(a_{1}\right)<a_{1} \text { and } f\left(a_{2}\right)>a_{2} \text {, }
$$

then $f$ has a fixed point in $\left[a_{1}, a_{2}\right]$. It is a natural question whether this result can be extended to the case of ordered Banach spaces. A number of fixed point theorems with assumptions of type (1) are well known; see, e.g., [1, Section 2.1]. However, to the best of our knowledge, fixed point theorems with assumptions of type (2) have not been known so far. In the present note, we prove the following fixed point theorem of this type.

Theorem 1 Let $X$ be a real Banach space with an order cone $K$ satisfying

(a) $K$ has a nonempty interior,

(b) $K$ is normal and minihedral.

Assume that there are two points in $X, u_{-} \ll u_{+}$, and a monotone increasing compact continuous operator $T:\left[u_{-}, u_{+}\right] \rightarrow X$. If $u_{-}$is a strong supersolution of $T$ and $u_{+}$is a strong subsolution, that is,

$$
T u_{-} \ll u_{-} \text {and } T u_{+} \gg u_{+} \text {, }
$$

then $T$ has a fixed point $u_{*} \in\left[u_{-}, u_{+}\right]$.

\section{Springer}

(C) 2012 Kostrykin and Oleynik; licensee Springer. This is an Open Access article distributed under the terms of the Creative Commons Attribution License (http://creativecommons.org/licenses/by/2.0), which permits unrestricted use, distribution, and reproduction in any medium, provided the original work is properly cited. 
Here $\left[u_{-}, u_{+}\right]$denotes the order interval $\left\{u \in X: u_{-} \leq u \leq u_{+}\right\}$.

Theorem 1 generalizes an idea developed by the present authors in [2], where the existence of solutions to a certain nonlinear integral equation of Hammerstein type has been shown.

Before we present the proof, we recall some notions. We write $u \geq v$ if $u-v \in K, u>v$ if $u \geq v$ and $u \neq v$, and $u \gg v$ if $u-v \in \stackrel{\circ}{K}$, where $\stackrel{\circ}{K}$ is the interior of the cone $K$.

A cone $K$ is called minihedral if for any pair $\{x, y\}, x, y \in X$, bounded above in order there exists the least upper bound $\sup \{x, y\}$, that is, an element $z \in X$ such that

(1) $x \leq z$ and $y \leq z$,

(2) $x \leq z^{\prime}$ and $y \leq z^{\prime}$ implies that $z \leq z^{\prime}$.

Obviously, a cone $K$ is minihedral if and only if for any pair $\{x, y\}, x, y \in X$, bounded below in order there exists the greatest lower bound $\inf \{x, y\}$. If a minihedral cone has a nonempty interior, then any pair $x, y \in X$ is bounded above in order. Hence, $\sup \{x, y\}$ and $\inf \{x, y\}$ exist for all $x, y \in X$.

A cone $K$ is called normal if there exists a constant $N>0$ such that $x \leq y, x, y \in K$ implies $\|x\|_{X} \leq N\|y\|_{X}$.

By the Kakutani-Krein brothers theorem [3, Theorem 6.6] a real Banach space $X$ with an order cone $K$ satisfying assumptions (a) and (b) of Theorem 1 is isomorphic to the Banach space $C(Q)$ of continuous functions on a compact Hausdorff space $Q$. The image of $K$ under this isomorphism is the cone of nonnegative continuous functions on $Q$.

An operator $T$ acting in the Banach space $X$ is called monotone increasing if $u \leq v$ implies $T u \leq T v$.

Consider the operator $\widehat{T}:\left[u_{-}, u_{+}\right] \rightarrow X$ defined by

$$
\widehat{T} u:=\sup \left\{\inf \left\{T u, u_{+}\right\}, u_{-}\right\} .
$$

Since $\inf \left\{T u_{+}, u_{+}\right\}=u_{+}$and $\sup \left\{u_{+}, u_{-}\right\}=u_{+}, u_{+}$is a fixed point of the operator $\widehat{T}$. Similarly, one shows that $u_{-}$is also a fixed point.

Lemma 2 The operator $\widehat{T}$ is continuous, monotone increasing, compact and maps the order interval $\left[u_{-}, u_{+}\right]$into itself.

Proof For any $v \in K$, the maps $u \mapsto \sup \{u, v\}$ and $u \mapsto \inf \{u, v\}$ are continuous; see, e.g., Corollary 3.1.1 in [4]. Due to the continuity of $T$, it follows immediately that $\widehat{T}$ is continuous as well. The operator $\widehat{T}$ is monotone increasing since inf and sup are monotone increasing with respect to each argument. Therefore, for any $u \in\left[u_{-}, u_{+}\right]$, we have

$$
u_{-}=\widehat{T} u_{-} \leq \widehat{T} u \leq \widehat{T} u_{+}=u_{+} \text {. }
$$

Let $\left(u_{n}\right)$ be an arbitrary sequence in $\left[u_{-}, u_{+}\right]$. Since $T$ is compact, $\left(T u_{n}\right)$ has a subsequence $\left(T u_{n_{k}}\right)$ converging to some $v \in X$. From the continuity of $\widehat{T}$, it follows that the sequence $\left(\widehat{T} u_{n_{k}}\right)$ converges to $\sup \left\{\inf \left\{v, u_{+}\right\}, u_{-}\right\}$, thus, proving that the range of $\widehat{T}$ is relatively compact.

Lemma 3 There exist $p_{ \pm} \in X$ with

$$
u_{-} \ll p_{-} \ll p_{+} \ll u_{+}
$$


and

$$
\widehat{T} p_{-}<p_{-}, \quad \widehat{T} p_{+}>p_{+} .
$$

Proof Due to $T u_{-} \ll u_{-}$, there is a $\delta>0$ such that $B_{\delta}\left(u_{-}-T u_{-}\right) \subset \stackrel{\circ}{K}$. The preimage of $B_{\delta}\left(u_{-}-T u_{-}\right)$under the continuous mapping $u \mapsto u-T u$ contains a ball $B_{\epsilon}\left(u_{-}\right)$. Hence, $u-T u \gg 0$ holds for all $u \in B_{\epsilon}\left(u_{-}\right)$. By the same argument, $u-T u \ll 0$ for all $u \in B_{\epsilon}\left(u_{+}\right)$. Choosing $\epsilon>0$ sufficiently small, we can achieve that $B_{\epsilon}\left(u_{-}\right) \cap B_{\epsilon}\left(u_{+}\right)=\varnothing$.

Set $p(t):=\left\{(1-t) u_{-}+t u_{+} \mid t \in[0,1]\right\}$. We choose $t_{-} \in(0,1)$ so small that $p_{-}:=p\left(t_{-}\right) \in$ $B_{\epsilon}\left(u_{-}\right)$and $t_{+} \in(0,1)$ so close to 1 that $p_{+}:=p\left(t_{+}\right) \in B_{\epsilon}\left(u_{+}\right)$. Then we have $u_{-} \ll p_{-} \ll$ $p_{+} \ll u_{+}$and

$$
T p_{-} \ll p_{-}, \quad T p_{+} \gg p_{+} .
$$

Due to $p_{-} \ll u_{+}$and $T p_{-} \ll p_{-}$, we have inf $\left\{T p_{-}, u_{+}\right\}=T p_{-}$. Further, we obtain

$$
\sup \left\{T p_{-}, u_{-}\right\} \leq \sup \left\{p_{-}, u_{-}\right\}=p_{-} .
$$

From $T p_{-} \ll p_{-}$it follows that there is an element $z \ll 0$ such that $T p_{-}=p_{-}+z$. Assume that $\sup \left\{T p_{-}, u_{-}\right\}=p_{-}$. Then we have $\sup \left\{z, u_{-}-p_{-}\right\}=0$. However, in view of the KakutaniKrein brothers theorem, $u_{-}-p_{-} \ll 0$ implies $\sup \left\{z, u_{-}-p_{-}\right\} \ll 0$. Thus, it follows that $\sup \left\{T p_{-}, u_{-}\right\} \neq p_{-}$and, therefore, $\widehat{T} p_{-}<p_{-}$. Similarly one shows that $\widehat{T} p_{+}>p_{+}$.

The main tool for the proof of Theorem 1 is Amann's theorem on three fixed points (see, e.g., [5, Theorem 7.F and Corollary 7.40]):

Theorem 4 Let $X$ be a real Banach space with an order cone having a nonempty interior. Assume there are four points in $X$,

$$
p_{1} \ll p_{2}<p_{3} \ll p_{4},
$$

and a monotone increasing image compact operator $\widehat{T}:\left[p_{1}, p_{4}\right] \rightarrow X$ such that

$$
\widehat{T} p_{1}=p_{1}, \quad \widehat{T} p_{2}<p_{2}, \quad \widehat{T} p_{3}>p_{3}, \quad \widehat{T} p_{4}=p_{4} .
$$

Then $\widehat{T}$ has a third fixed point $p$ satisfying $p_{1}<p<p_{4}, p \notin\left[p_{1}, p_{2}\right]$, and $p \notin\left[p_{3}, p_{4}\right]$.

Recall that the operator is called image compact if it is continuous and its image is a relatively compact set.

We choose $p_{1}=u_{-}, p_{2}=p_{-}, p_{3}=p_{+}, p_{4}=u_{+}$, where $p_{ \pm}$is as in Lemma 3 . Since the cone $K$ is normal, by Theorem 1.1.1 in [1], $\left[u_{-}, u_{+}\right]$is norm bounded. Thus, $\widehat{T}$ is image compact.

Theorem 4 yields the existence of a fixed point $u_{*}$ of the operator $\widehat{T}$ satisfying $u_{-}<u_{*}<u_{+}$. Obviously, $u_{*}$ is a fixed point of the operator $T$ as well. This observation completes the proof of Theorem 1 . 
Authors' contributions

All authors contributed equally. All authors read and approved the final manuscript

\section{Author details}

'FB 08 - Institut für Mathematik, Johannes Gutenberg-Universität Mainz, Staudinger Weg 9, Mainz, D-55099, Germany.

${ }^{2}$ Current address: Department of Mathematics, University of Uppsala, P.O. Box 480, Uppsala, S-75106, Sweden.

\section{Acknowledgements}

The authors thank H.-P. Heinz for useful comments. This work has been supported in part by the Deutsche Forschungsgemeinschaft, Grant KO 2936/4-1.

Received: 5 June 2012 Accepted: 5 November 2012 Published: 22 November 2012

\section{References}

1. Guo, D, Lakshmikantham, V: Nonlinear Problems in Abstract Cones. Academic Press, Boston (1988)

2. Kostrykin, V, Oleynik, A: On the existence of unstable bumps in neural networks. Preprint. arXiv:1112.2941 [math.DS] (2011)

3. Krasnosel'skij, MA, Lifshits, JA, Sobolev, AV: Positive Linear Systems. The Method of Positive Operators, Sigma Series in Applied Mathematics, vol. 5. Heldermann, Berlin (1989)

4. Chueshov, I: Monotone Random Systems Theory and Applications. Lecture Notes in Mathematics, vol. 1779. Springer, Berlin (2002)

5. Zeidler, E: Nonlinear Functional Analysis and Its Applications: I: Fixed-Point Theorems. Springer, New York (1986)

doi:10.1186/1687-1812-2012-211

Cite this article as: Kostrykin and Oleynik: An intermediate value theorem for monotone operators in ordered

Banach spaces. Fixed Point Theory and Applications 2012 2012:211.

\section{Submit your manuscript to a SpringerOpen ${ }^{\circ}$ journal and benefit from:}

- Convenient online submission

- Rigorous peer review

- Immediate publication on acceptance

Open access: articles freely available online

- High visibility within the field

- Retaining the copyright to your article 